former days the bird-life appears, however, to have been even more abundant than at the present day, this being especially the case with regard to kittiwakes, which were once found in thousands where there are now hundreds. So numerous, indeed, were these birds that there is a record of the heaps of twitch left in a field on a Saturday to be carted on the Monday having been carried off in the meantime by the gulls for nest building. The usual ruthless massacres of the old day's were, however, responsible for so reducing the numbers of these birds that they were wellnigh exterminated by the time the Protection Acts once more gave them a chance.

After referring briefly to the puffin and the razorbill, accompanying his notice of the former by an excellent figure of a young bird (herewith reproduced), the author treats in considerable detail of the breeding habits and eggs of the guillemot. Attention is called to the number of young ones and eggs which are destroyed by falling down the cliffs when the birds are suddenly frightened, the author expressing his belief that a guillemot will intentionally roll its egg from the ledge on which it rests if she thinks it is about to be carried off. The remarkable variation displayed by guillemot eggs naturally claims a share of attention, although the author confesses that he is unable to give any reason for the phenomenon. In this connection it may be

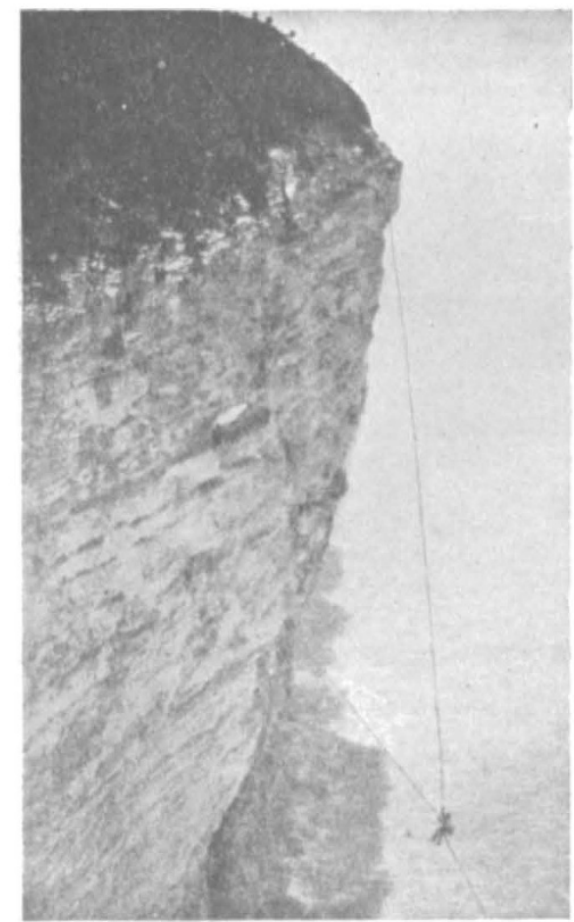

Fig. 2.-A descent in search of eggs. (From the "Birds ut Be upion Cliffs.")

mentioned that a magnificent series of these eggs, showing nearly all the chief types of variation, has recently been placed on exhibition in the Natural History Museum.

Cliff-climbing in Yorkshire is always effected by means of ropes, the author describing it as the most delightful and exciting form of gymnastics. Judging from the illustration here reproduced, some of our readers might think it a trifle too exciting. At the present time from 300 to 400 eggs are collected daily during the season, the total take being about 130,0no. In spite of this drain the numbers of the birds annually increase. The price of the eggs varies from twelve to sixteen a shilling, abnormally marked specimens fetching from $2 d$. to $7 s$. $6 d$., or even more, each. R. L.

\section{A NEW BINOCULAR.}

A NEW form of prismatic binocular, styled the " Service," has recently been issued by Messrs. J. H. Dallmeyer, and there are many interesting features about it for which special advantages may be claimed. As a rule, binoculars consist of two independent optical trains in separate coverings, either hinged together to allow for the different gauges between the separation of human eyes, or made in different sizes to suit these various distances. In the present form the whole optical arrangement is enclosed in one cover, and in consequence of this, six out of the eight prisms employed and the two objective sliding tubes can all be fixed rigidly to one frame, thus ensuring maintenance of adjustment and strength in adverse circumstances.

The separation of the eye-pieces is secured by a screw adjustment situated between them, by which each eye-piece with one prism slides in strong grooves in a lateral direction. For any one individual this adjustment is constant, so that when once the correct position has been attained a permanent mark can be made, and this position quickly regained at any other time; the maximum separation between the centres of the eye-pieces is $70 \mathrm{~mm}$. and the minimum $55 \mathrm{~mm}$., so plenty of latitude is available for abnormal eyes.

There is another screw adjustment for the ordinary focusing, and one of the eye-pieces can be separately adjusted by mearls of a graduated spiral movement in case the observer's eyes are not similar. All these different manipulations can be easily made when only one hand is available, and the whole mechanism can be fully exposed for cleaning the optical surfaces by simply taking out four screws which in no way interfere with any of the adjustments.

Constructed chiefly of magnalium, and in parts of gun-

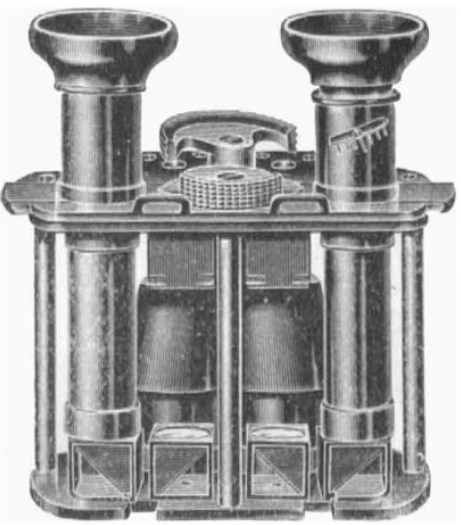

metal, the glasses are light in weight, and it is claimed that they are smaller, power for power, than any other prismatic glass yet made. There are five sizes on the market varying in magnifying power from four to twelve times, the former weighing thirteen and the latter sixteen ounces; the smaller sizes are suitable for theatre or night use.

\section{SEISMOLOGICAL NOTES.}

THE last publication of the Earthquake Investigation Committee of Japan, issued this year, is of special interest to those engaged in seismometry. In it Prof. A. Tanakadate describes a vertical motion seismometer, in which a mass is so suspended that it is not affected by tilting or by horizontal shocks, and remains in neutral equilibrium for vertical displacements of considerable magnitude. Until this instrument was devised, for large earthquakes at least, vertical spring seismographs, and for that matter horizontal bracket seismographs, have responded to the changes in inclination of their supports, with the result that they have

NO. 1742 , voL. 67$]$ 